\title{
Impact on the quality of life of patients suffering from osteoarthritis of the knee after intra-articular administration of bone marrow mononuclear cells
}

\author{
Marcus Valadares Guimarães ${ }^{1 *}$, Dalton Lopes Terra ${ }^{1}$, José Fabio Santos Duarte Lana ${ }^{1}$ and Paula Alexandra da Graça Morais ${ }^{2}$ \\ ${ }^{1}$ Orthopedist, Member of Brasilian Society of Orthopedic and Traumatology, Brazil \\ ${ }^{2}$ Biologist, Federal University of Juiz de Fora, MG, Brazil
}

\begin{abstract}
Objective: The aim of this study was to evaluate the combined treatment of BMMCs alone or in combination of arthroscopic debridement and lavage in treatment of knee OA through resonance image and quality of life questionnaire. In comparison to the mesenchymal stem cells (MSC), the effectiveness is lower, however the costs for manipulation and laboratory handling make it difficult to use in clinical practice.

Design: This was a pilot, longitudinal and prospective trial with two years of follow-up. Twenty-one patients with patellofemoral osteoarthritis who met the study criteria were included. The patients were divided into three groups: BMMCs+Arthroscopy; BMMCs+lavage; BMMC. Clinical outcomes were evaluated using SF-36 questionnaire at baseline and two years after the procedure.

Results: In this study, it was verified a high recovery of BMMCs and platelets, especially with manual separation. It was not observed differences in SF-36 when comparing the three groups, however the evaluation of SF-36 baseline and two years of follow-up in each one of the three groups, it was showed an improvement in 3-4 parameters. The MRI showed an improvement in the stroke, subchondral bone and cartilage size in the patella and femur.

Conclusion: This study demonstrated that OA showed a significant improvement, measured by quality life questionnaire, only with the use of BMMCs, showing no improvement with the combination of arthroscopy or joint lavage. In this way, the use of BMMC's is well accepted, a presented impact on parameters of SF-36. None of the patients underwent total knee arthroplasty. A study with a higher number of patients is of great value to assess the safety and efficacy of BMMC's application.
\end{abstract}

\section{Introduction}

Articular cartilage has a limited intrinsic capacity to regenerate spontaneously after injury, often leading to pain and disability. It is generally believed that cartilage lesions progress to osteoarthritis (OA). OA of the knee is one of the most chronic degenerative joints diseases, affecting the quality of life of patient. Prompt intervention for symptomatic lesions make possible prevention of evolution to OA as well as to provide symptom relief. Conventional treatment modalities may be useful for relief of symptoms in the short term; however, they do not restore the natural articular cartilage integrity or prevent the deterioration [1]. In addition, the surgery for knee replacement provides a solution for severe OA [2]. The conservative nonsurgical treatments include analgesics, nonsteroid and steroid anti-inflammatory drugs and corticosteroids $[3,4]$. When the conservative treatment fails to control the symptoms and functional limitations occur, surgery should be considered to treat the cartilage lesion and the anatomical abnormalities. Conventional methods used to regenerate anormalies of the articular cartilage include microfractures, multiple perforation, abrasion and mosaicoplasty, with limited results [5].

Orthobiologics is a thriving area of research and development, aimed specifically at preventing further degeneration and disease by restoring native biology, structure, and function. Cell-based therapy is a form of regenerative medicine that introduces new cells to repair damaged tissue [1]. Nowadays, there are a variety of orthobiologics such as: whole blood therapy, traditional prolotherapy, platelet rich plasma (PRP), autologous conditioned plasma (ACP) or autologous conditioned serum, bone marrow aspirate, adipose biocellular autografts, allograft of mesenchymal stem cells are the most wellstudied and prevalent grafts of current use [6]. In this study we focus on autologous mononuclear cells obtained from bone marrow (BMMC). In cell therapy, the majority of studies have used mesenchymal stem cells derived from bone marrow (BMSC). It's important to note that, the BMMCs, enriched with BMSCs have shown to be beneficial [7]. Previous clinical trials have demonstrated beneficial effects in osteonecrosis of femoral head, relieving pain and prevents the progression of osteonecrosis. The number of cells used increased 3 folds basal number, reaching $35.2 \times 10^{6}$ cells $/ \mathrm{mL}$ [8]. We published a study evaluating the use of BMMCs and arthroscopy to treat patellofemoral osteoarthritis and verified promising results, reducing signs of patellofemoral $\mathrm{AO}$ and ensure the patient satisfaction with a safe return to social life and sports and improvement in functional scores, restoring the articular cartilage of subchondral bone [9]. However, the use of BMMCs for OA is not

${ }^{\star}$ Correspondence to: Marcus Valadares Guimarães, Nucleus of Orthopedics and Traumatology (NOT) Rua Aimorés 2125, Belo Horizonte, Minas Gerais, Brazil, Tel: +55 31 3349-2600; Fax: +55 31 3349-2638; E-mail mvg@ortopediamarcusvguimaraes.com

Key words: patellofemoral joint, osteoarthritis, bone marrow mononuclear cells

Received: March 27, 2019; Accepted: April 08, 2019; Published: April 12, 2019 
well explorate in the literature. In this way, the aim of this study was to evaluate the combined treatment of BMMCs alone or in combination of arthroscopic debridement, lavage in treatment of knee OA through resonance image and quality of life questionnaire.

We believe that combined treatment including arthroscopic debridement, lavage and local administration of the autologous fraction of bone marrow mononuclear cell (BMMC) or only an injection of BMMC, can improve the articular function and the life quality of the patients through a simple, safe procedure with low morbidity rate.

\section{Material and methods}

\section{Research design}

This was a pilot, longitudinal and prospective study with two years of follow-up. After a review and approval by the institutional ethics committee, the volunteer participants did a cell collection and received a treatment by the interventionist. Clinical and radiological evaluations were made before the procedure and six months later. Questionnaire of life quality, the Short Form 36 (SF-36) were used. These evaluations were made at baseline and two years after the procedure. The grade of osteoarthritis was verified through the the Kellgreen-Lawrence classification. The patients were divided into three groups: Group BMMC+Arthroscopy, Group lavage+BMMCs and Group BMMC.

\section{Patients}

The study was conducted in the Nucleus of Orthopedics and Traumatology, in Belo Horizonte, MG, Brazil from june of 2012 to february of 2014. It was evaluated 21 patients, and all of them were enrolled in the study. After obtaining approval from the Belo Horizonte Orthopedics and Traumatology Center Ethics Committee (authorization number 001/2012) and the required patient informed consent, the patients with a positive diagnostic of arthritis obtained from magnetic resonance image (MRI) and radiological evidence of the knee were selected for treatment. The criteria for inclusion in the study were: $30-85$ years of age, negative results and non-reactive to rheumatic autoimmune disease and a diagnostics of osteoarthritis (OA) obtained with MRI. The grades of OA according to Kellgreen-Lawrence was moderate to severe (III and/or IV). The patients were instructed not to take anti-inflammatory drugs 30 days before and during the procedure. The exclusion criteria were: a 5-year malignity diagnostics prior to the procedure; pregnancy or breast-feeding; active neurological disease; uncontrolled endocrine disorders (diabetes, hypothyroidism); active cardiac condition or respiratory disease dependent on medication; positive or reactive tests for syphilis; Chagas disease; B, C, or HIV1+2 and HTLV1+2 hepatitis serological markers [10].

Patients were assessed at screening for clinical and demographic characteristics and baseline assessment. They complete a questionnaire (SF-36) in baseline and two years after the procedure. Radiology was made six months after the procedure.

\section{BMMC collection and preparation}

For sample removal, the patient was placed on a prone position Criovida ${ }^{\oplus}$ operating table. After the proper asepsis of the pelvis, the posterior superior iliac crest was anesthetized with $20 \mathrm{ml}$ of $1 \%$ lidocaine. Four $20 \mathrm{~mL}$ syringes were filled with $1 \mathrm{~mL}$ heparina $(5000$ UI) each. Puncture aspiration of the area anesthetized was performed with an Osgood-type myelogram needle. After being filled, the syringes were properly capped and homogenized. It was collected an average of $58.44 \mathrm{~mL}$ bone marrow material (varying from 39 to $87 \mathrm{~mL}$ ) from each patient. The samples were kept in cold storage and prepared between 12 -18 hours after the collection.

For the 8 patients ( 8 knees), the BMMC were sorted and isolated using a Ficoll ${ }^{\circ}$ method (Ficoll ${ }^{\circ}$ Paque Plus Ge 17-1440-02 Healthcare) and a Sepax ${ }^{\oplus}$ separator CS-900.2 - Biosafe - Switzerland. The BMMCs were washed and then placed into a sterile syringe resuspending in saline solution with $20 \%$ of albumin. For the other 13 patients ( 25 knees), samples were manually prepared with centrifuges Jouan (GR422). It was used the Ficoll Paque Plus GE 17-1440-02 (Ge Healthcare) for mononuclear isolation, following the manufacter's instruction. An aliquot of the prepared samples was separated for analysis in the cell conter KX21 (Sysmex). The first eight patients used the Sepax ${ }^{\oplus}$ system because it is what we had we had in stock in the laboratory. For the other patients we chose the manual system due to financial constraints. All the patients received only one application of BMMCs.

\section{Patients treatment}

Eight patients (11 knees) underwent arthroscopy for joint debridement under sedation. It was used a tourniquet at the root of the limb with an average level of $300 \mathrm{mmHg}$. The knee was washed with an Arthrex ${ }^{\oplus}$ pump filled with saline sterile solution and pressure used was $40 \mathrm{mmHg}$. The lateral and medial infrapatellar were evaluated. The debridement consisted of the removal of cartilage fragments, free bodies and debridement of cartilage lesions. The mean procedure time was 15 minutes. The knee was punctured by arthroscopic vision confirming the presence of the intra-articular needle. A physiological withdrawal was performed with $1250 \mathrm{~mL}$ of sterile saline solution, after it was removed from an arthroscopy apparatus and injected $10 \mathrm{~mL}$ of BMMCs. Six patients ( 10 knees) underwent knee lavage under sedation with sterile saline solution $(2000 \mathrm{ml}$ of final volume). Suprapatellar lateral and medial infrapatellar punctures were performed, and the knee washed using a $60 \mathrm{ml}$ syringe. After withdrawal of the saline solution, 10 $\mathrm{ml}$ of BMMCs was injected. Seven patients (12 knees) underwent intraarticular injection of $10 \mathrm{~mL}$ of BMMCs in the knee in a clean area in the office. The patient's group was chosen in random way, according to the chronological order that they were eligible for the study.

\section{Results}

\section{Casuistic}

Twenty-one patients were included in the study: seven men and fourteen women. Average age was 62 years (39-85 years of age). Twelve patients had a both knees compromised; nine had a only one knee compromised (six right and three left). Seven patients had only a injection of BMMC into their knees and 8 had arthroscopic surgery for debridement and an injection of BMMC. The other six patients received a wash with saline and the BMMCs application. All patients had previously undergone a six months conservative treatment with the administration of non-hormonal anti-inflammatory drugs, physiotherapy treatment and condroitin/glicosamin. All patients refused a total knee replacement (arthroplasty). Six patients had a body mass index less than 30 , and fifteen greater than 30 . According to Kellgreen-Lawrence scale, eight patients were classified as grade 3 and thirteen patients as grade 4 . The casuistic of the patients were described in Table 1.

\section{BMMCs contents}

The average of volume harvested from bone marrow in manual and commercial procedure of BMMCs (and maximum and minimum 
values), administered volume and the absolute number of cells (BMMCs and platelets) were described in the Table 2 .

\section{Clinical evaluation-life quality-SF-36}

The comparison of SF-36 among the groups showed no difference in none of the parameters in basal and two years after the procedure. However, when evaluate the treatment group basal and after two years of the procedure, it was verified a significant improvement in some parameters of the SF-36. For BMMC's+arthroscopy it was showed a significant improvement in 4 parameters: functional skills, physical limitations, social skills and emotional skills. For the group of $\mathrm{BMMC}^{\prime}$ s+lavage it was demonstrated a significant improvement in 3 parameters: functional skills, social skills and pain. For the group of BMMC's it was verified a significant improvement in 5 parameters: functional skills, physical limitations, pain, vitality and emotional skills, as shown in the Table 3.

When comparing the results of the SF-36 among 8 patients with whom Sepax had been used and 13 patients with whom had been done manual separation, no difference was shown between groups $(\mathrm{p}=0.854)$.

Patients were asked how they felt about the results and whether they would repeat the treatment or not in the future. Three patients reported that they had no improvement with treatment and would not repeat. These three patients were: 78 and 80 years old from the group of BMMCs, and 86 years old from the group of BMMCs+arthroscopy. These three patients had OA degree 4 on the Kellgreen-Lawrence scale. However, the other three patients over 75 years of age had good results. Eighteen were happy with the treatment and would consider repeating.
On analysis of MRI and X-rays, all patients showed changes in the exam. All were classified as grade 3 (8 patients) and grade 4 (13 patients). Findings such as stroke, osteophytes, tricompartimental joint space decrease, signal increase in the subchondral bone in the femur, patella and / or tibia and loose bodies, were common. After 6 months the MRI was repeated. There was no change in the presence of osteophytes, tricompartimental joint space decrease or loose bodies. The stroke decreased in 15 patients and remained the same in 6 patients. Ten patients showed improved cartilage size in the patella and femur. The most common finding was the standard improvements the subchondral bone. The three patients who reported no improvement with the treatment, maintained the subchondral bone changes such as increased focal signal translating osteochondral fractures and subchondral edema in T2. The 18 patients who reported improvement showed no sign of increase in the subchondral bone in $\mathrm{T} 2$.

There were no adverse reactions, like local infections or complaints about the intra-articular administration of BMMC. No patient underwent total knee arthroplasty.

\section{Discussion}

An OA is the most common joint disease and the major cause of disability in the adult population; with annual costs of knee OA being immense, this continues to be a severe health burden when it comes to morbidity and expense. Age is the primary OA risk factor, and aging-related changes also contribute to pathophysiological changes triggering OA disease. In addition, individuals with other specific OA risk factors, including obesity, altered joint mechanical loading, joint injury and inflammation, as well as genetic components, may experience an accelerated rate of changes that are like those associated

Table 1. Description of patients' characteristics

\begin{tabular}{|c|c|c|c|c|}
\hline Group & Gender M:F & Median age (years) & OA Grade & Knees \\
\hline \multirow[t]{2}{*}{ Arthroscopy + BMMC } & 02:06 & $57(37-86)$ & $62.5 \% \mathrm{IV}$ & $62.5 \%$ Unilateral \\
\hline & & & $37.5 \%$ III & $37.5 \%$ Bilateral \\
\hline \multirow[t]{2}{*}{ Lavage + BMMC } & 00:06 & $44.5(37-76)$ & $33.3 \%$ IV & $33.3 \%$ Unilateral \\
\hline & & & $66.7 \%$ III & $66.7 \%$ Bilateral \\
\hline \multirow[t]{2}{*}{ BMMC } & 02:05 & $69(54-80)$ & $85.7 \%$ IV & $28.6 \%$ Unilateral \\
\hline & & & $14.3 \% \mathrm{III}$ & 71.4\% Bilateral \\
\hline P value & & n.s. & n.s. & \\
\hline
\end{tabular}

Table 2. Characteristics of BMMCs used into the joints. Sepax ${ }^{\circledR}$ (closed method), Manual (open method)

\begin{tabular}{|c|c|c|c|c|}
\hline & $\begin{array}{l}\text { Volume } \\
\text { collected } \\
(\mathrm{ml})\end{array}$ & $\begin{array}{l}\text { Final volume } \\
\text { administered } \\
\text { (ml) }\end{array}$ & $\begin{array}{c}\text { Total BMMC } \times 10^{6} \\
(\text { cels } / \mathrm{uL})\end{array}$ & $\begin{array}{c}\text { Total platelets } \times 10^{6} \\
(\text { cels } / \mathbf{u L})\end{array}$ \\
\hline Sepax average & $\begin{array}{c}58.44 \\
(39-87)\end{array}$ & 10 & $\begin{array}{c}147.75 \\
(73-310)\end{array}$ & $\begin{array}{c}562.75 \\
(260-1000)\end{array}$ \\
\hline Manual average & $\begin{array}{c}46.48 \\
(26-100)\end{array}$ & 10 & $\begin{array}{c}279.84 \\
(29.4-680)\end{array}$ & $\begin{array}{c}1715.8 \\
(140-9000)\end{array}$ \\
\hline
\end{tabular}

Table 3. Life quality of patients according to the different groups

\begin{tabular}{|c|c|c|c|c|c|c|c|c|c|}
\hline Group & Time & $\begin{array}{l}\text { Functional } \\
\text { Skills }\end{array}$ & $\begin{array}{c}\text { Physical } \\
\text { Limitations }\end{array}$ & Pain & $\begin{array}{c}\text { General Health } \\
\text { State }\end{array}$ & Vitality & Social Skills & $\begin{array}{c}\text { Emotional } \\
\text { Skills }\end{array}$ & Mental Health \\
\hline \multirow[t]{2}{*}{ BMMCs+Arthroscopy } & Basal & $28.8 \pm 26.4$ & $9.4 \pm 18.6$ & $39 \pm 28.7$ & $60 \pm 7.6$ & $57.5 \pm 20.7$ & $46.9 \pm 28.1$ & $25 \pm 34.5$ & $53.5 \pm 18.4$ \\
\hline & 2 years & $68.8 \pm 31.1$ & $50 \pm 48.2$ & $63.5 \pm 17.3$ & $63.7 \pm 11.3$ & $65.6 \pm 17.4$ & $76.7 \pm 24$ & $70.8 \pm 41.5$ & $70 \pm 18.1$ \\
\hline$P$ value & & $0.0139 *$ & $0.0238 *$ & n.s. & n.s. & n.s. & $0.0295^{*}$ & $0.0363 *$ & n.s. \\
\hline \multirow[t]{2}{*}{ BMMCs+lavage } & Basal & $15 \pm 14.8$ & $25 \pm 41.8$ & $32.5 \pm 21.1$ & $72.5 \pm 6.9$ & $46.7 \pm 24$ & $60.4 \pm 24.3$ & $27.8 \pm 39$ & $58.7 \pm 22.6$ \\
\hline & 2 years & $65 \pm 33.8$ & $58.3 \pm 46.5$ & $62.3 \pm 21.2$ & $72.5 \pm 6.9$ & $65 \pm 20.5$ & $77.1 \pm 26.7$ & $77.8 \pm 34.4$ & $66 \pm 18.2$ \\
\hline$P$ value & & $0.0067^{*}$ & n.s. & $0.0304 *$ & n.s. & n.s. & $0.0429 *$ & n.s. & n.s. \\
\hline \multirow[t]{2}{*}{ BMMCs } & Basal & $18.6 \pm 17.7$ & $28.6 \pm 48.8$ & $42.7 \pm 20$ & $72.9 \pm 7$ & $57.1 \pm 19.5$ & $69.6 \pm 21.5$ & $38.1 \pm 44.8$ & $67.4 \pm 20$ \\
\hline & 2 years & $60.7 \pm 31.7$ & $46.4 \pm 39.3$ & $71.6 \pm 18.8$ & $72.1 \pm 8.1$ & $71.4 \pm 15.5$ & $83.9 \pm 17.2$ & $85.7 \pm 26.2$ & $72 \pm 16$ \\
\hline P value & & $0.0076 *$ & $0.0465 *$ & $0.0310 *$ & n.s. & $0.0465 *$ & n.s. & $0.0465 *$ & n.s. \\
\hline
\end{tabular}


with aging [11,12]. Although cartilage destruction is the hallmark of OA, and collagen erosion is the pivotal event that determines the irreversible progression of $\mathrm{OA}$ disease, it is now well established that $\mathrm{OA}$ is not only a disorder of cartilage homeostasis but is a whole-joint disorder involving all joint tissues, including the subchondral bone, menisci and synovial membrane [13,14]. In spite of recent advances, the mechanisms leading to cartilage destruction in patients with $\mathrm{OA}$ are still not clearly identified and no successful therapeutic intervention exists.

Synovitis cause pain, cartilage degradation and pannus formation with subsequent erosions. It is increasingly recognized that synovitis is also observed both in early and in late OA. Indeed, synovitis predicts structural severity and progression of tibiofemoral cartilage damage in OA [15]. Histological features of OA synovitis include synovial lining hyperplasia, infiltration of macrophages and lymphocytes, angiogenesis and fibrosis [14]. Subchondral inflammation might also contribute to increased bone turnover and joint damage in OA [16]. Mechanical factors and specific immunity being key to knee OA progression, common mechanisms may contribute to joint damage and pain. Macrophage infiltration is a characteristic feature of synovitis and is associated with radiographic joint damage in OA [14].

Subchondral bone turnover is increased in OA, as evidenced by bone formation and resorption biomarkers [17], as well as by imaging techniques, including radiography [18], computerized tomography, magnetic resonance imaging [19], dual X-ray absorptiometry and scintigraphy [20]. Another interesting finding was the size reduction of edematous subchondral spots, evidenced in the MRI and referenced in Emadedin et al. Those effects may have resulted from the antiinflammatory impact of the MSC as mentioned in previous studies [21]. In our study we found MRI images which suggest an improvement of symptoms is related to improvements of subchondral bone, regardless of cartilage recover.

Recent research suggests the increasing importance of subchondral bone integrity in various orthopedic conditions including osteoarthritis. Bone marrow lesions seen on T2 MRI sequences in osteoarthritic patients demonstrate histology similar to nonunion fractures with necrosis and high osteoclast activity and are becoming an important biomarker in disease progression $[21,22]$.

Mesenchymal stem cells are a promising therapy for cartilage regeneration. The exact mechanism of action of mesenchymal stem cells is not completely understood, but various means have been proposed. Through paracrine activity, mesenchymal stem cells exhibit a secretory or "trophic" function, with anti-inflammatory, immunomodulatory, pro-angiogenic, anti-apoptotic, anti-fibrotic, and wound-healing properties that have proliferative effects [23]. Mesenchymal stem cells have been shown to elicit differentiation of resident and nonresident cells to functional tissue, catalyzing restoration of degenerative tissue. It has been suggested that perivascular cells, or pericytes, adhere to blood vessels and act as 1 of our body's largest reservoirs for mesenchymal stem cells. After trauma, soluble factors within the perivascular space cause the release of pericytes from microvessels. Pericytes have been described as "medicinal signaling cells" once released, where they can be activated into mesenchymal stem cells, exhibiting their homing, trophic and immunomodulatory roles [24]

Xia et al. accessed the efficacy of mesenchymal stem cells (MSCs) injection in the treatment of knee OA in meta-analysis. They included randomized controlled and controlled clinical trials of people with knee
OA comparing the outcomes of pain and function for those receiving MSCs injection with those receiving no MSCs injection. Seven randomized controlled and controlled clinical trials, studying a total of 314 participants with a diagnosis of knee OA were included. But results from two high quality trials (94 patients) show a positive effect of MSCs injection on pain. Heterogeneity observed between studies regarding the effect of MSCs injection on pain and function was explained by the difference of follow-up time, outcome measures, control group, the source and dose of MSCs. The quality of evidence supporting these effect estimates was rated as low. The conclusion was MSCs injection could be potentially efficacious for decreasing pain and may improve physical function in patients with knee OA [25].

In relation to the BMMC's, there is no difference between Sepax ${ }^{\circ}$ systems and open system with the use of centrifuge as described, but the number of open cells in the system is increased. However, both systems have an appropriate number of cells. The choice between the systems should take the safety and cost into account [26].

The SF-36 study showed a significant improvement in various parameters of SF-36 in comparison of baseline and two years after treatment, especially with the use of only cells (BMMC group). The BMMC group showed a significant improvement in 5 parameters after two years of application, showing a good maintence of these cells. Also, as no difference was observed between the treatments, and the application of cells without other type of procedure promoted a great improvement in the patients, verified through the SF-36 questionnaire, shows that no other type of intervention is necessary in patients with $\mathrm{OA}$ of knee. Only the cells were able to promote promising and interesting results in OA patients, and there was no superior improvement when applied together with arthroscopy or joint lavage. The limitations of this study includes the low number of patients and evaluation of a quality life questionnaire, without a functional questionnaire for evaluate the impact of OA in patient's life.

\section{Conclusion}

This study demonstrated that OA showed a significant improvement, measured by quality life questionnaire, only with the use of BMMCs, showing no improvement with the combination of arthroscopy or joint lavage. In this way, the use of BMMC's is well accepted, a presented impact on parameters of SF-36. None of the patients underwent total knee arthroplasty. A study with a higher number of patients is of great value to assess the safety and efficacy of BMMC's application.

\section{References}

1. Sampson S, Bemden AB, Aufiero D (2015) Stem cell therapies for treatment of cartilage and bone disorders? osteoarthritis, avascular necrosis and non-union fractures. PM and $R$ 7: 26-32. [Crossref]

2. Carr AJ, Robertsson O, Graves S, Price AJ, Arden NK, et al. (2012) Knee replacement Lancet 379: 1331-1340. [Crossref]

3. Bijlsma JW, Berenbaum F, Lafeber FP (2011) Osteoarthritis: an update with relevance for clinical practice. Lancet 377: 2115-2126. [Crossref]

4. Mcalindon TE, Bannuru RR, Sullivan MC, Arden NK, Berenbaum F, et al. (2014) OARSI guidelines for the non-surgical management of knee osteoarthritis. Osteoarthr Cartil 22: 363-88.

5. Noyes FR, Barber-westin SD (2013) Advanced patellofemoral cartilage lesions in patients younger than 50 years of age? Is there an ideal operative option?? Arthrosc $J$ Arthrosc Relat Surg 29: 1423-1436.

6. Crane DM, Oliver KS, Bayes MC ((2016) Orthobiologics and knee osteoarthritis: A recent literature review, treatment algorithm and pathophysiology discussion. Phys Med Rehabil Clin 27: 985-1002. 
Guimarães MV (2019) Impact on the quality of life of patients suffering from osteoarthritis of the knee after intra-articular administration of bone marrow mononuclear cells

7. Song F, Tang J, Geng R, Hu H, Zhu C, et al. (2014) Comparison of the efficacy of bone marrow mononuclear cells and bone mesenchymal stem cells in the treatment of osteoarthritis in a sheep model. Int J Clin Exp Pathol 7: 1415-1426. [Crossref]

8. Wei BW, Shi SZ, Guo NZDYW, Li SXJLZ (2010) Treatment of nontraumatic osteonecrosis of the femoral head with the implantation of core decompression and concentrated autologous bone marrow containing mononuclear cells. Arch Orthop Trauma Surg 130: 859-865. [Crossref]

9. Guimarães MV, Alexandra P, Rios M, Ruiz MA, Noronha CP, et al. (2018) Patellofemoral osteoarthritis? treatment with autologous bone marrow mononuclear cells and arthroscopic surgery: A prospective study Stem Cell \& Regenerative Medicine 2: $2-7$.

10. Kaplan JM, Youd ME, Lodie TA (2011) Immunomodulatory activity of mesenchymal stem cells. Curr Stem Cell Res Ther 6: 297-316. [Crossref]

11. Sandell LJ (2012) Etiology of osteoarthritis? genetics and synovial joint development. Nat Publ Gr 8: 77-89.

12. Lotz M, Loeser RF (2012) Effects of aging on articular cartilage homeostasis. Bone 51: 241-248.

13. Heinegård D, Saxne T (2010) The role of the cartilage matrix in osteoarthritis. Nat Publ Gr 7: 50-56.

14. Scanzello CR, Goldring SR (2012) The role of synovitis in osteoarthritis pathogenesis? Bone 51: 249-257.

15. Ayral XMD, D EHPP, Woodworth TGMD, Mackillop NMD, Dougados MMD (2005) Synovitis? a potential predictive factor of structural progression of medial tibiofemora knee osteoarthritis e results of a 1-year longitudinal arthroscopic study in 422 patients. Osteoarthritis Cartilage 13: 361-367. [Crossref]

16. Walsh DA, Mcwilliams DF, Turley MJ, Dixon MR, Mapp PI, et al. (2010) Angiogenesis and nerve growth factor at the osteochondral junction in rheumatoid arthritis and osteoarthritis. Rheumatology 49: 1852-1861. [Crossref]
17. Rousseau JC, Garnero P (2012) Biological markers in osteoarthritis. Bone 51: 265 277.

18. Woloszynski T, Podsiadlo P, Stachowiak GW, Kurzynski M, Lohmander LS, et al. (2012) Prediction of progression of radiographic knee osteoarthritis using tibial trabecular bone texture. Arthritis Rheum 64: 688-695. [Crossref]

19. Hunter DJ, Harvey W, Gross KD, Felson D, Mccree P, et al. (2011) A randomized tria of patellofemoral bracing for treatment of patellofemoral osteoarthritis. Osteoarthritis Cartilage 19: 792-800. [Crossref]

20. Roman-blas JA, Castañeda S, Largo R, Lems WF, Herrero-beaumont G (2014) An OA phenotype may obtain major bene fi t from bone-acting agents. Semin Arthritis Rheum 43: 421-428.

21. Emadedin M, Aghdami N, Taghiyar L, Fazeli R, Moghadasali R, et al. (2012) Intraarticular injection of autologous mesenchymal stem cells in six patients with knee osteoarthritis. Arch Iran Med 15: 422-428. [Crossref]

22. Kraus VB, Feng S, Wang S, White S, Ainslie M, et al. (2013) Subchondral bone trabecular integrity predicts and changes concurrently with radiographic and magnetic resonance imaging - determined knee osteoarthritis progression. Arthritis Rheum 65 1812-1821. [Crossref]

23. Gupta PK, Das AK, Chullikana A, Majumdar AS (2012) Mesenchymal stem cells for cartilage repair in osteoarthritis. Stem Cell Res Ther 3: 25. [Crossref]

24. Caplan AI (2015) Adult mesenchymal stem cells? When, where and how. Stem Cells Int. 628767 [Crossref]

25. Xia P, Wang X, Lin Q, Li X (2015) Efficacy of mesenchymal stem cells injection for the management of knee osteoarthritis: a systematic review and meta-analysis. Int Orthop 39: 2363-2372. [Crossref]

26. Jo CH, Lee YG, Shin WH, Kim H, Chai JW, et al. (2014) Intra-articular injection of mesenchymal stem cells for the of osteoarthritis of the knee: a proof-of-concept clinical trial. Stem Cells 32: 1254-1266. [Crossref]

Copyright: (C2019 Guimarães MV. This is an open-access article distributed under the terms of the Creative Commons Attribution License, which permits unrestricted use, distribution, and reproduction in any medium, provided the original author and source are credited. 